\title{
Prevalencia e incidencia de discapacidad a partir del Certificado Único de Discapacidad en un hospital universitario del Área Metropolitana de Buenos Aires Prevalence and incidence of disability based on the Unique Certificate of Disability at a teaching hospital in the Metropolitan Area of Buenos Aires
}

\author{
Dra. Celeste Puga ${ }^{a}$, Dra. Vanina Pagotto ${ }^{b}$ Dr. Diego Giunta ${ }^{b}$, Dra. Jimena Vicens ${ }^{b}$, Dra. Mariana Leist ${ }^{a}$, \\ Dr. Esteban Vaucheret Paz ${ }^{a}$, Dra. Lucila Hornstein ${ }^{c}$, Dr. Leonardo Garfic y Dr. Guillermo Agosta ${ }^{a}$
}

\section{RESUMEN}

La discapacidad es un problema de salud pública que afecta las oportunidades de desarrollo integral del individuo. El objetivo del trabajo fue estimar la prevalencia e incidencia anual y de las categorías diagnósticas asociadas a discapacidad total y por grupos etarios a partir de la tramitación del Certificado Único de Discapacidad.

Estudio analítico de una cohorte de niños/as de 0 a 18 años perteneciente a un hospital universitario del Área Metropolitana de Buenos Aires, entre enero de 2010 y diciembre de 2017.

Sobre un total de 22750 afiliados activos, 726 pacientes tramitaron elCertificadoÚnico de Discapacidad; la prevalencia fue del 3,2 \% (IC 95\%:2,9-3,4). La incidencia acumulada anual aumentó desde 2012 (0,22\%; IC 95\%:0,1-0,19) hasta 2017 (0,59\%; IC $95 \%: 0,5-0,7)$. Las discapacidades mentales constituyeron el $80 \%(\mathrm{n}=576)$.

En este estudio se observó un aumento de la incidencia de discapacidad y de la categoría de discapacidad mental. Palabras clave: personas con discapacidad, epidemiología, trastorno del espectro autista, discapacidad intelectual, pediatría.

http: / / dx.doi.org/10.5546/ aap.2019.183

Texto completo en inglés:

http:/ / dx.doi.org/10.5546/ aap.2019.eng.183

Cómo citar: Puga C, Pagotto V, Giunta D, Vicens J, et al. Prevalencia e incidencia de discapacidad a partir del Certificado Único de Discapacidad en un hospital universitario del Área Metropolitana de Buenos Aires. Arch Argent Pediatr 2019;117(3):183-187.

a. Departamento de Pediatría, Servicio de Neurología del Hospital Italiano de Buenos Aires.

b. Departamento de Investigación, Hospital Italiano de Buenos Aires.

c. Gerencia de Plan de Salud del Hospital Italiano de Buenos Aires.

Correspondencia:

Dra. Celeste Puga: celeste.puga@hospitalitaliano.org.ar

Financiamiento: Ninguno.

Conflicto de intereses: Ninguno que declarar.

Recibido: 12-6-2018

Aceptado: 5-12-2018

\section{INTRODUCCIÓN}

Según el "Informe Mundial sobre la Discapacidad", 95 millones de niños en todo el mundo presentan alguna discapacidad, de los cuales 13 millones (el 0,7 \%) tienen una discapacidad grave. ${ }^{1}$ En nuestro país, la Encuesta Nacional de Personas con Discapacidad (ENDI) de 2003 estimó que el $7 \%$ de los habitantes del país vivía con alguna discapacidad, de los cuales 254497 eran niños / as menores de 15 años (el $4 \%)^{2,3}$

La Clasificación Internacional del Funcionamiento, de la Discapacidad y de la Salud (CIF) define la discapacidad como un término genérico que abarca deficiencias, limitaciones de la actividad y restricciones a la participación. ${ }^{4}$ Se entiende por discapacidad la interacción entre las personas que padecen alguna enfermedad (por ejemplo, parálisis cerebral, síndrome de Down y depresión) y factores personales y ambientales (por ejemplo, actitudes negativas, transporte y edificios públicos inaccesibles y un apoyo social limitado).

Si bien las investigaciones sobre discapacidad utilizan el marco conceptual de la CIF, existen diferencias en los datos relacionados con la prevalencia, con el tipo de preguntas que se utilizan, con su orden y con el marco muestral usado. $^{1,5}$

En nuestro país, las personas con discapacidad se encuentran en condiciones de solicitar de manera voluntaria el Certificado Único de Discapacidad (CUD). ${ }^{6}$

El objetivo del estudio fue estimar la prevalencia e incidencia anual y de las categorías diagnósticas asociadas a discapacidad total y por grupos etarios a partir de la tramitación del CUD y del requerimiento de apoyos.

\section{MÉTODOS}

Diseño: Estudio analítico, observacional, de una cohorte de niños / as de 0 a 18 años a partir de 
registros médicos electrónicos del Plan de Salud del Hospital Italiano de Buenos Aires (PS-HIBA), desde enero de 2010 a diciembre de 2017.

Población: Se incluyeron los menores que presentaban CUD o discapacidad con necesidad de soportes, entendidos como los recursos terapéuticos, dispositivos de asistencia o estrategias utilizadas para que las personas con discapacidad desarrollaran su máxima potencialidad en entornos comunitarios. Se clasificaron los pacientes en 4 grupos etarios (0-4 años, 5-9 años, 10-14 años, 15-18 años).

Los diagnósticos fueron agrupados en 4 categorías. Estas eran similares a las utilizadas en los censos y a la clasificación utilizada en el CUD, que utilizaba diagnósticos de acuerdo con el Manual Diagnóstico y Estadístico de los Trastornos Mentales (DSM IV) y con el Manual para la Clasificación Internacional de Enfermedades (CIE 10): mental (incluyó a niños con trastorno del espectro autista-TEA-, discapacidad intelectual, retraso global del desarrollo, trastorno del aprendizaje, trastorno del lenguaje y trastornos del comportamiento), motora (parálisis cerebral, enfermedades neuromusculares, enfermedades del tubo neural), sensorial (hipoacusia y ceguera) y visceral (cardiopatías, enfermedad fibroquística del páncreas, síndrome de intestino corto).

\section{Análisis estadístico}

La prevalencia de período (2010-2017) total y por grupo etario se calculó utilizando la prevalencia ponderada anual de afiliados activos menores de 18 años, considerando la edad a mitad del período en estudio. La incidencia acumulada anual de discapacidad total y por grupo etario se calculó considerando como denominador el total de afiliados activos menores de 18 años a la mitad de cada año. Se estimaron los intervalos de confianza (IC) del $95 \%$ para las incidencias y las prevalencias. Las variables continuas se expresaron como medianas con su respectivo rango intercuartilo (RIC) del 25-75 \%. Las proporciones fueron expresadas como frecuencias absolutas y relativas. El protocolo fue aprobado por el Comité de Ética de Protocolos de Investigación del HIBA.

\section{RESULTADOS}

En el período de estudio, 726 pacientes menores de 18 años, sobre un total de 22750 afiliados activos al PS-HIBA, tramitaron

FIGURA 1. Incidencia acumulada global anual de discapacidad cada 100 afiliados con su intervalo de confianza del $95 \%$

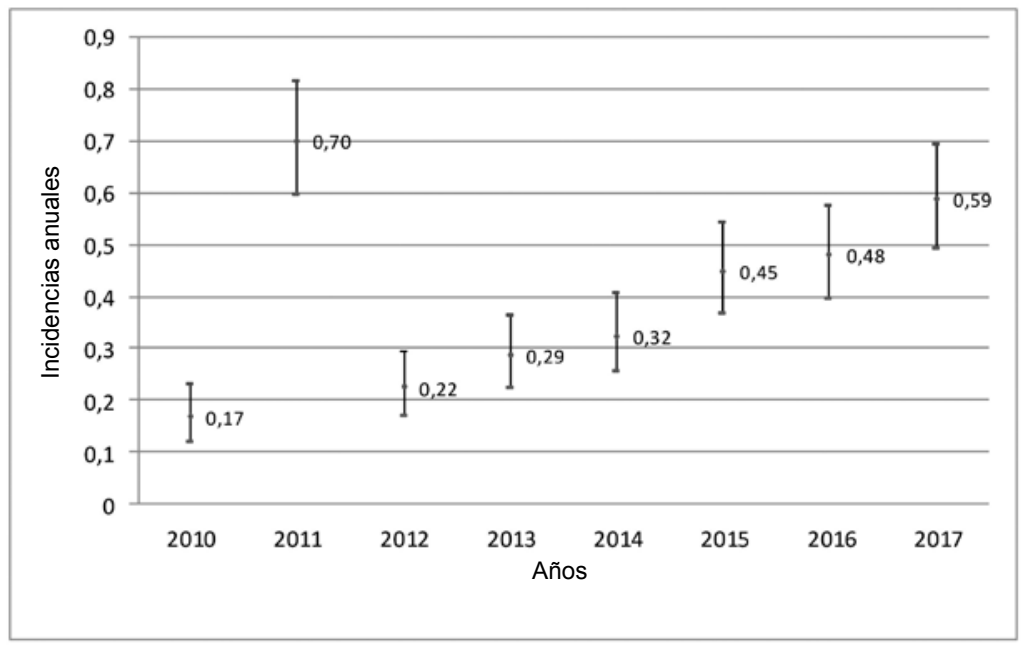

TABLA 1. Incidencia acumulada anual de discapacidad por grupo etario cada 100 afiliados con intervalo de confianza del $95 \%$

\begin{tabular}{lcccccccc}
\hline Grupo etario & $\mathbf{2 0 1 0}$ & $\mathbf{2 0 1 1}$ & $\mathbf{2 0 1 2}$ & $\mathbf{2 0 1 3}$ & $\mathbf{2 0 1 4}$ & $\mathbf{2 0 1 5}$ & $\mathbf{2 0 1 6}$ & $\mathbf{2 0 1 7}$ \\
\hline 0-4 años & 0 & 0 & 0 & $0,1(0,06-0,2)$ & $0,06(0,02-0,2)$ & $0,4(0,3-0,7)$ & $0,6(0,5-0,9)$ & $0,9(0,7-1,1)$ \\
5-9 años & $0,09(0,04-0,2)$ & $0,3(0,2-0,5)$ & $0,4(0,2-0,5)$ & $0,4(0,28-0,6)$ & $0,6(0,5-0,9)$ & $0,5(0,4-0,8)$ & $0,6(0,5-0,9)$ & $0,9(0,7-1,1)$ \\
10-14 años & $0,4(0,25-0,59)$ & $1,5(1,3-2)$ & $0,3(0,2-0,5)$ & $0,4(0,3-0,6)$ & $0,3(0,2-0,6)$ & $0,5(0,4-0,7)$ & $0,3(0,2-0,5)$ & $0,2(0,1-0,3)$ \\
15-18 años & $0,3(0,17-0,58)$ & $1,5(1,14-2)$ & $0,2(0,1-0,5)$ & $0,08(0,03-0,2)$ & $0,1(0,06-0,34)$ & $0,1(0,04-0,3)$ & $0,1(0,07-0,3)$ & $0,2(0,1-0,5)$ \\
\hline
\end{tabular}


el CUD o requirieron algún soporte terapéutico, que resultó en una prevalencia de período del 3,2 \% (IC $95 \%: 2,9-3,4 \%$ ). La mediana de edad de los pacientes fue 9 años (RIC: 5-12); el $65 \%$ fueron varones $(\mathrm{n}=478)$. El $68 \%$ de los pacientes $(\mathrm{n}=496)$ pertenecían al grupo de 5 a 14 años. El $91 \%(\mathrm{n}=662)$ contaba con CUD, mientras que el $9 \%(\mathrm{n}=64)$ restante recibía algún tipo de soporte terapéutico sin la tramitación de dicho certificado.

En el grupo etario comprendido entre 0 y 4 años, la prevalencia fue del $2 \%$ (IC $95 \%$ : 1,72,4\%); en el grupo de 5-9 años, del 3,8 \% (IC $95 \%$ : 3,4-4,3\%); en el grupo de 10-14 años, del $4 \%$ (IC $95 \%: 3,5-4,5 \%)$, y, finalmente, en el grupo de 15 a 18 años, del 2,8\% (IC $95 \%$ : 2,3-3,4\%). En la Figura 1, se muestra la incidencia global por año y, en la Tabla 1, las incidencias acumuladas anuales y por grupo etario.

En cuanto a las categorías diagnósticas, el $80 \%(\mathrm{n}=576)$ presentaba discapacidad mental. El $20 \%$ restante $(\mathrm{n}=150)$ presentaba discapacidad no mental: el $9 \%$, motora $(\mathrm{n}=64)$; el $7 \%$ $(\mathrm{n}=54)$, sensorial, y el $5 \%$, visceral $(\mathrm{n}=32)$. El $19 \%$ presentaba 2 o más discapacidades (el
$9 \%$, mental-motor; el $6 \%$, mental-visceral, y el $1 \%$, mental-sensorial). Véase la Tabla 2. De los 576 pacientes con discapacidad mental, el $71 \%$ $(\mathrm{n}=405)$ eran varones. En la Figura 2, se observa la incidencia acumulada anual de discapacidad mental y no mental en el período en estudio. La proporción de pacientes con diagnóstico de discapacidad mental aumentó del $56 \%$ en 2010 a un $88 \%$ en 2017, y no se observaron cambios en la pirámide poblacional a lo largo de todo el período. Dentro de la discapacidad mental, los diagnósticos más frecuentes fueron discapacidad intelectual (el $37 \%, \mathrm{n}=212$ ), que incluía a niños con síndrome de Down (el $18 \%, \mathrm{n}=38$ ), y TEA (el $40 \%, \mathrm{n}=234)$, y, entre ambos diagnósticos, se constituyó el $77 \%$ del total de esa categoría.

\section{DISCUSIÓN}

La prevalencia correspondiente al período 2010-2017 de la población de niños/as pertenecientes al PS-HIBA fue del 3,2\%, que resultó menor que la reportada en la ENDI ${ }^{2}$ (que la ubicaba en un $4 \%$ ) y que la del Censo de $2010^{7}$ (del $4,8 \%$ ), y cercana a la informada por la

TABLA 2. Información complementaria de diagnósticos asociados

\begin{tabular}{ll}
\hline & $\mathrm{N}(\%)$ \\
\hline Discapacidad mental & 576 \\
Trastorno del espectro autista & $234(40)$ \\
Discapacidad intelectual & 212 \\
$\quad$ Síndrome de Down & $38 / 212$ \\
$\quad$ Otros síndromes genéticos (Prader-Willi, Sotos, Klinefelter) & $10 / 212$ \\
$\quad$ Encefalopatías epilépticas & $3 / 212$ \\
$\quad$ Otros diagnósticos & $11 / 212$ \\
$\quad$ Sin diagnóstico etiológico & $150 / 212$ \\
Trastorno del lenguaje & $39(7)$ \\
Trastorno del aprendizaje - TDAH - trastornos del comportamiento & $77(13)$ \\
Otros diagnósticos & $14(3)$ \\
Discapacidad motora & $64(8)$ \\
Parálisis cerebral & $31 / 64$ \\
Mielomeningocele - espina bífida & $7 / 64$ \\
Neuropatías - miopatías & $9 / 64$ \\
Otros (accidentes - ACV - tumores del SNC) & $17 / 64$ \\
Discapacidad sensorial & $54(7)$ \\
Hipoacusia & $52 / 54$ \\
Discapacidad visceral & $32(4)$ \\
Cardiopatías & $5 / 32$ \\
Fibrosis quística & $5 / 32$ \\
Síndrome de intestino corto - colitis ulcerosa - enfermedad de Crohn - trasplante de hígado & $9 / 32$ \\
Otras & $13 / 32$ \\
\hline
\end{tabular}

TDAH: trastorno por déficit de atención e hiperactividad; ACV: accidente cerebrovascular; SNC: sistema nervioso central. 
Encuesta Anual de Hogares (EAH), que arrojó una prevalencia en el mismo grupo etario del 3,1 \%. ${ }^{8}$ Resulta difícil comparar los datos obtenidos tanto en la ENDI como en el Censo de 2010 y en la EAH, debido a que, si bien se encuentran diseñadas bajo el marco teórico propuesto por la $C I F$, la información fue recolectada de diferentes maneras. ${ }^{1}$

Para esta investigación, se utilizó el dato proveniente de los soportes requeridos, con o sin CUD, y se observó que, en su mayoría, los pacientes poseían CUD. En nuestro país, se estima que solo el $14,6 \%$ de las personas con discapacidad cuentan con CUD..$^{9,10}$ De ellas, el $23 \%$ son niños/as menores de 14 años, es decir, más del $70 \%$ de los/as niños / as con discapacidad no tienen CUD. ${ }^{9}$ La diferencia observada en comparación con los resultados obtenidos en nuestro trabajo puede responder a múltiples factores. Núñez y colaboradoras mencionan, en su trabajo, que los familiares manifestaron falta de información en cuanto a los asuntos legales y desconocimiento de los derechos básicos, como la obtención del CUD o de pensiones. ${ }^{11}$ Para la tramitación del CUD, es necesaria la consulta con una/un especialista en Psiquiatría o en Neurología Infantil, aspecto que, en algunas comunidades, resulta difícil de llevar a cabo.

En cuanto a las incidencias acumuladas estimadas en cada año del período de estudio, se observó un marcado aumento en 2011, que podría verse asociado a la sanción de la Ley $\mathrm{N}^{\mathrm{o}} 26682$ (Marco Regulatorio de Medicina Prepaga), que establecía la obligatoriedad de cubrir, como mínimo, lo dispuesto en el Programa Médico Obligatorio..$^{12,13}$
En cuanto al tipo de discapacidad, se halló que, en su mayoría, los pacientes presentaban como diagnóstico principal discapacidad mental. Los datos publicados en 2011 por el Censo Nacional reportaron, en cambio, que el $35,1 \%$ tenía una limitación visual y solo el $26,3 \%$ cognitiva. ${ }^{14}$ Solo en combinación con otras discapacidades, el déficit cognitivo resultó más frecuente. Se cree que las diferencias observadas se deben al tipo de preguntas realizadas en el Censo para caracterizar las distintas discapacidades. ${ }^{14}$ La predominancia de varones con diagnóstico de discapacidad mental fue notablemente mayor, hallazgo que coincidió con otros trabajos de prevalencia e incidencia de trastornos del desarrollo. ${ }^{15}$

A partir del análisis de la incidencia anual por categoría diagnóstica, se observó que las discapacidades sensoriales, motoras y viscerales se mantuvieron sin mayores modificaciones a lo largo de todo el período 2010-2017. Sin embargo, la categoría correspondiente a discapacidad mental, que incluyó diagnósticos como discapacidad intelectual, TEA y trastornos en el comportamiento, mostró un incremento sostenido a lo largo del período. Es posible que este sea el resultado de una mejor vigilancia y un mayor conocimiento por parte de los padres y madres, así como de los profesionales médicos que realizan los cuidados en salud de los niños. ${ }^{16}$

Dentro de las limitaciones del trabajo, se puede mencionar que la definición de discapacidad se basó en la necesidad de soportes (tratamiento psicológico, psicopedagógico, kinesiología, integración escolar, dispositivos ortopédicos o implantes) con o sin la tramitación del CUD, por lo que podría encontrarse subestimado

FIGURA 2. Incidencias acumuladas por año, por cada 100 afiliados según categoría diagnóstica mental o no mental

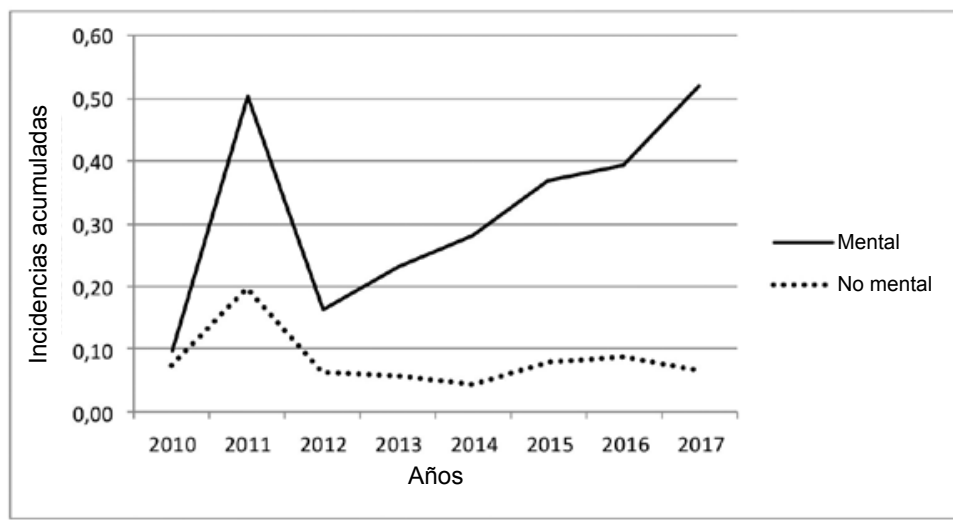


el total de niños / as con alguna discapacidad, al no considerar a aquellos que, aun con una discapacidad, no tenían CUD ni recibían dicho soporte. Asimismo, al tratarse de una población perteneciente a un sistema de medicina privado residente en su mayoría en el Área Metropolitana de Buenos Aires, los resultados encontrados podrían ser diferentes a los encontrados en el resto del país.

La discapacidad es un problema de salud pública que afecta las oportunidades de desarrollo integral del individuo y lo vuelve vulnerable, debido, principalmente, a las limitaciones de accesibilidad a la salud y a la educación. En el presente trabajo, se observó un aumento en la incidencia de discapacidad acompañado de un incremento de la categoría de discapacidad mental. Conocer los datos de prevalencia e incidencia, así como los diagnósticos asociados a la discapacidad, permite el armado de programas de intervención y atención orientados a mejorar la calidad de vida y la inclusión social de los / as niños/as con discapacidad.

\section{REFERENCIAS}

1. Organización Mundial de la salud. Informe Mundial Sobre la Discapacidad. Ginebra: OMS; 2011. [Consulta: 6 de diciembre de 2018]. Disponible en: https: / / www.who. int/disabilities/world_report/2011/es/.

2. Rodríguez Gauna MC. Primera Encuesta Nacional de Personas con Discapacidad (ENDI) de Argentina. $\mathrm{Su}$ potencialidad para el estudio de la población con discapacidad. [Consulta: 6 de diciembre de 2018]. Disponible en: http:// repositoriocdpd.net:8080/ handle/123456789/358.

3. INDEC Direcciones Provinciales de Estadísticas. Aquí se cuenta. Revista informativa del censo 2001 2004:14. [Consulta: 28 de abril 2018]. Disponible en: https: / / www.indec.gov. ar/micro_sitios/webcenso/aquisecuenta/aqui14.pdf.

4. OrganizaciónMundialdelaSalud.Clasificacióninternacional del funcionamiento de la discapacidad y de la salud: CIF: versión abreviada. Madrid: Grafo; 2001. [Consulta: 6 de diciembre de 2018]. Disponible en: http:/ / apps.who.int/ iris / bitstream / handle / 10665 / 43360/9241545445_spa. pdf?sequence $=1$.

5. Joly, E. ¿Podemos confiar en la ENDI?. IV Jornadas Nacionales Universidad y Discapacidad. Reconocer la
Diferencia para Proteger la Igualdad. Del 5 al 7 de julio de 2006. Buenos Aires: Facultad de Derecho; 2006. [Consulta: 2 de junio de 2018]. Disponible en: http: / / www.rumbos. org.ar/podemos-confiar-en-la-endi.

6. Agencia Nacional de Discapacidad. Certificado Único de Discapacidad. [Consulta: 2 de junio de 2018]. Disponible en: https://www.argentina.gob.ar/como-obtener-elcertificado-unico-de-discapacidad-cud.

7. INDEC. Censo Nacional de Población, Hogares y Viviendas 2010. Resultasos de la consulta: Cuadros (5). Buenos Aires, Argentina, 2011. [Consulta: 2 de junio de 2018]. Disponible en: https://www.indec.gob.ar/ censos_total_pais.asp?id_tema_1=2\&id_tema_2=41\&id_ tema $3=135 \& \mathrm{t}=3 \& \mathrm{~s}=2 \& \mathrm{c}=2010$.

8. Pantano L, Rodríguez Gauna MC. La población con dificultad de largo plazo en la Ciudad Autónoma de Buenos Aires (Informe1). Encuesta Anual deHogares 2011. Buenos Aires: Dirección General de Estadísticas y Censo; 2013. Disponible en: https:/ /www.estadisticaciudad.gob.ar/ eyc/wp-content/uploads/2015/04/2011_poblacion_con_ dificultad_largo_plazo.pdf.

9. Moya G. Derechos de los pacientes con enfermedades que se asocien con discapacidad: marco legal actual. Arch Argent Pediatr. 2016; 114(4):355-60.

10. INDEC ECMI. Censo 2001 - Encuesta Nacional Complementaria de Personas con Discapacidad. [Acceso: 6 deoctubre de2018]. Disponibleen:http:/ / www.indec.gov. ar/micro_sitios/webcenso/ENDI_NUEVA/ampliada_ lista_total.asp?Cap=13\&Apertu=0.

11. Pantano L, Nuñez B, Arenaza A. ¿Qué Necesitan Las Familias de Personas Con Discapacidad? Investigación, reflexiones y propuestas. Buenos Aires: Lugar Editorial; 2012.

12. Ley 26682. Marco Regulatorio de Medicina Prepaga. Buenos Aires, Argentina, 16 de mayo de 2011. [Consulta: 1 de junio de 2018]. Disponible en: http://fepra.org.ar/feprav3/ documentos/normas_ejercicio_pais / Ley-de-medicinaprepaga_26682.pdf.

13. Superintendencia de Servicios de Salud. Ministerio de Salud. Programa Médico Obligatorio (PMO). Resolución 201/2002. [Consulta: 2 de junio de 2018]. Disponible en: https:/ / www.sssalud.gob.ar/pmo/res_s_02_201.pdf.

14. INDEC. Censo Nacional de Población, Hogares y Viviendas 2010. Población Con Dificultad o Limitación Permanente. Octubre de 2014. [Consulta: 2 de junio de 2018]. Disponible en: https://www.indec.gov.ar/ftp/cuadros/sociedad/ PDLP_10_14.pdf.

15. Yeargin-Allsopp M, Rice C, Karapurkar T, Doernberg N, et al. Prevalence of Autism in a US Metropolitan Area. JAMA. 2003; 289(1):49-55.

16. BroscoJ, Mattingly M,Sanders L. Impact of Specific Medical Interventions on Reducing the Prevalence of Mental Retardation. Arch Pediatr Adolesc Med. 2006; 160(3):302-9.

El peor enemigo del conocimiento no es la ignorancia, es la ilusión del conocimiento. 